\title{
Analysis of Human Factors for Enhancing Safety and Security Management System in Fossil and Renewable Power Plants
}

\author{
Mohamed Younes El Bouti*, Mohamed Allouch \\ Faculty of Sciences and Technology of Tangier, Abdelmalek Essaâdi University, Ziaten, BP: 416, 90000 Tangier, Morocco
}

Corresponding Author Email: elbouti.mohamedyounes@etu.uae.ac.ma

https://doi.org/10.18280/ijsse.100408

Received: 28 May 2020

Accepted: 10 July 2020

\section{Keywords:}

accidents prevention, countermeasures, human factors (HFs), safety and security management system (SSMS), power plants (pps), safety-related behavior ( $S-R B)$

\begin{abstract}
While Safety and Security Management System (SSMS) in the energy industry has recently improved in the last decades, it remains a hazardous working environment where fatalities and serious accidents still reoccur. Human Factors (HFs) and worker SafetyRelated Behavior (S-RB) have been identified as the underlying causes of the majority of occurred accidents. Hence, this work aimed to identify those HFs which affect the SSMS from the most to the least significant, through the impact rate of these factors on workers S-RB at workplaces of twenty-one power plants (PPs), including Fossil Fuel Power Plants (FFPPs) and Onshore Wind Farms (OWFs) that are located across nine countries. Likewise, to give adequate countermeasures for the SSMS enhancement and accidents prevention. To do so, the data were collected through the survey questionnaire of a fivepoint Likert scale. The study has led us to conclude that, all the analyzed HFs have influenced the SSMS of all the evaluated PPs with an impact rate of 3.3/5 on Likert scale. Whereas the enhancement of the SSMS can be achieved if the job factors are improved and if the employees' "Workload" is well managed at the workplace, as well as if the management clearly demonstrates their H\&S commitment and leadership.
\end{abstract}

\section{INTRODUCTION}

Energy allows us to travel, study and works in the evenings and at nights, uses our smartphones and computers, drives our cars, lights up the cities and rural areas and so forth. All of these are the daily human activities, which become an integral part of their routine life, whereas, any disturbance in term of energy availability, certainly the situation will affect the people life quality and their continuous development. Thereby, electricity has become one of the crucial elements in daily human activities to have a normal life.

Occupational incidents and accidents form a social phenomenon and considered as one of the big problems worldwide. Although all the applied Health and Safety (H\&S) controls in any firm, as well as, the enhancement in occupational safety which have been known nowadays, things can still go wrong, as results accidents and incidents still reoccur. Statistics revealed that in every 15 seconds, one employee dies from work accidents, and 153 employees have an occupational accident. Moreover, 6,300 employees die daily as a result of occupational work-related accidents or illhealth [1].

The work-related accidents rates have raised alongside the industrial revolution and the fast world globalization. As a result, all sectors suffer from occupational adverse events at the workplace, particularly, at hazardous working environment such as the energy industry. Behind the comfortable life that the energy industry provides to the human society and the boost that it gives to the country's economy, the negative side that this sector knows is that, is deemed as one of the most suffered sectors from the mishaps [2, 3]. Fritzsche [3] emphasized that about $25 \%$ of the fatalities caused by severe mishaps around the world in the period 19701985 took place in the energy field. Therefore, the energy industry has been known as one of the main contributors to human-made disasters.

However, people in favor of fast industry development have not always been conscious of environmental degradation and human suffering that may arise afterwards. Many previous researches substantiated that over than half of the work accidents due to the poor S-RB of the workers and the failure of human behavior in the workstations $[4,5]$. This means that, if the workers behavior is overlooked and not considered during developing and implementing the SSMS, this will lead to raising behavioral non-compliance with Occupational Safety and Health $(\mathrm{OSH})$ requirements by the workers [6] Wherefore, the frequency and seriousness of work-related accidents which occur all over the world, particularly in the energy sector will stay highly astounding in terms of human and property losses [2] if the accidents underlying cause not tackled effectively in advance. This asserts that a good S-RB of the worker contributes to a good process safety in the workplace; consequently, it reduces the accidents occurrence and benefits both in time and cost-saving [7]. Therefore, the main goal of this study is to to tackle the safety and security concern in the hazardous working environment (Energy sector) to determine the critical factors which affect the SSMS in the energy firms.

This study is expected to shed new light on the development of the S-RB model for Moroccan and worldwide setting in the energy industry by tackling the HFs that affect the worker safety behaviour. Particularly, Morocco has alarming statistics in terms of occupational accidents [8-10].

The study addresses two kinds of energy power generation 
(OWFs and FFPPs) involving developing and developed countries. This will permit to compare the results between national and international experience as well as, between fossil fuel industry and wind industry in order to assist in finding out the commonalities of factors which affect the SSMS and identify effective control measures to enhance the SSMS in the energy industry as a whole or in a particular energy sector (Fossil or wind energy plants). In the same vein, this study gives the possibility to employ the findings in improving the SSMS in the wind and fossil PPs in developing countries similar to Morocco where the energy sector is still growing. Former study substantiated that the developing countries are extremely suffering from a high percentage of occupational mishaps than in the developed countries [11] where many of them are underreported $[9,12]$. Similarly, the international standards and working procedures do not focus on the HFs when developing and implementing the desired H\&S procedures and processes [13]. Since the HFs affect the workers S-RB, which is difficult to manage in the upstream phase (Before accident) when they are still actions and behaviour. However, the development of a positive and safe behaviour of the employees as a result of the SSMS improvement is expected to facilitate the operation of energy plants and reduce negative safety outcomes (Workplace accidents).

\section{FACTORS INFLUENCING THE SSMS}

\subsection{What is the role of the human factors in the SSMS?}

The HFs are the most critical elements in ensuring the effectiveness of the SSMS. This latter mainly relies on the adoption and implementation of OSH rules by everyone from senior management to lowest-ranking employees [14]. When looking at the causes of the majority of the occurred accidents were mainly consisting of HFs such as human errors (HEs) and violations of those implemented OSH rules, standards, policies and procedures. In other words, if the workers are trained with these requirements and well know them, it doesn't mean that they will also follow them. It noteworthy to state that the risk of human failure has a significant influence on SSMS in the workplace, which leads to an increase in the probability of occupational accidents. Therefore, the risk of human intervention is higher in a hazardous installation such as power generation plants.

The Figure 2 show how the HFs components which are considered as "Performance Shaping Factors" [15] affects the SSMS via S-RB of the employees in the workplace. In other words, the HFs can affect the worker behavior positively when promoting safe practices, and workers comply with safety requirements in the workstation, safety topic receives higher priority than other business issues, HEs decrease significantly, good safety culture and so forth. Thus, safety compliance achieved, and the employees demonstrate the desired safety performance in the workplaces, which leads to diminishing the likelihood of mishaps [16]. As well as, the HFs aspects can affect the safety and security performance negatively via the employee S-RB when promoting unsafe practices, workers do not comply with safety requirements in the workstation, safety topic receives lower priority than other business issues, poor safety culture, and so forth. Figure 1 illustrate how the HFs can be considered as a trigger of human behavior, which can be positive or negative behavior, depending on the inputs.

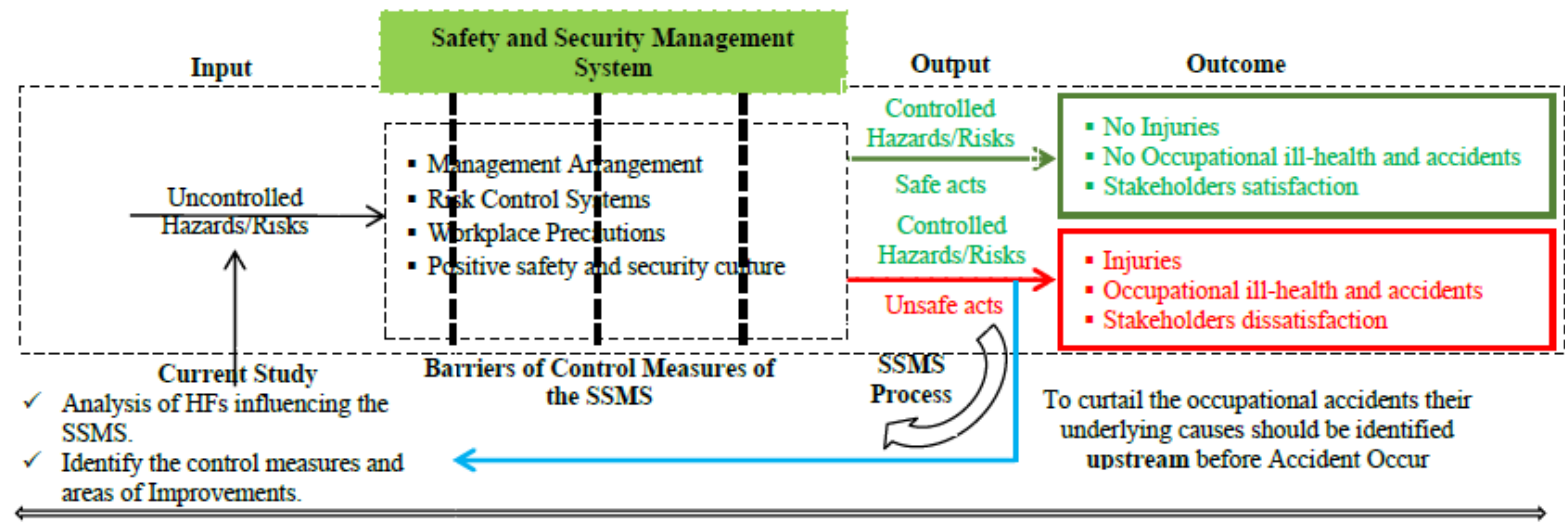

Figure 1. The impact of the human factors in improving the SSMS

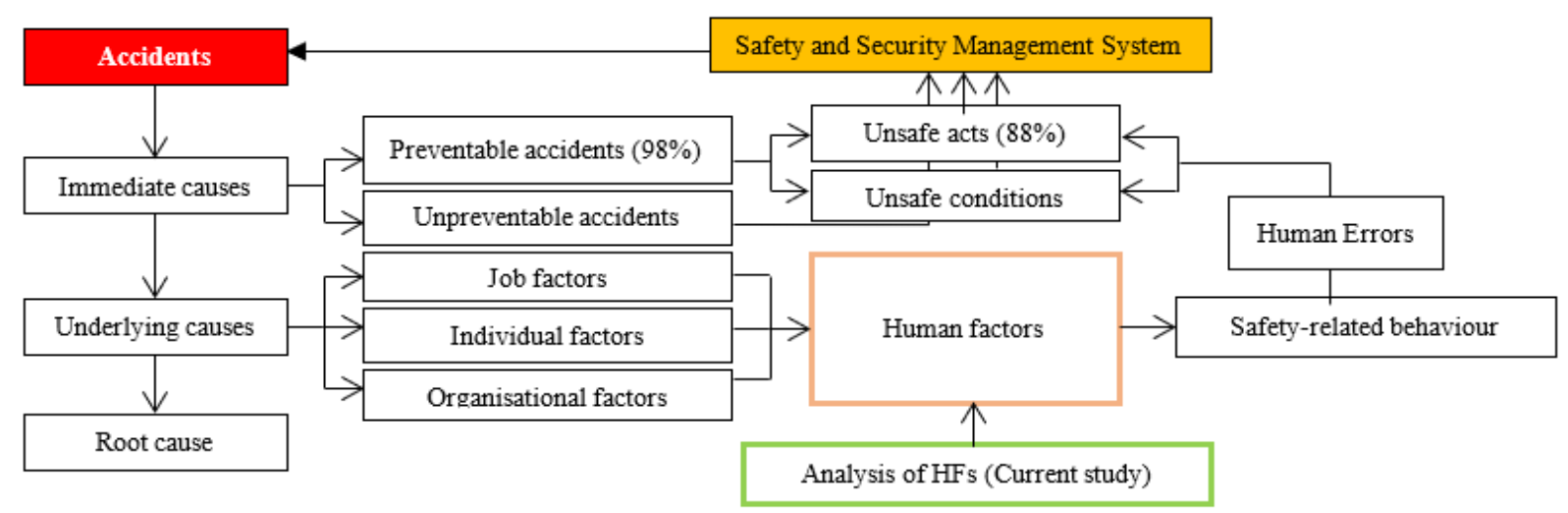

Figure 2. Human factors affect the SSMS leading to workplace accidents 


\subsection{Why human factors are influencing the SSMS?}

Human factors are a broad field and firms may have seen it in the past as being too hard or complex to do anything about it. Although the contribution of human failure to accidents are widely recognized, very few firms proactively addressed potential problems of human performance. Likewise, attributing accidents to human errors has often been a sufficient explanation in itself and something which is beyond the control of managers. Consequently, the HFs are overlooked during the accident investigation as a result the firm do not learn from the occurred incidents in order to bring adequate control measures to eliminate the underlying cause (HFs) and avoid recurrence. Yet, Figure 2 show how the HFs remain always affecting workplace safety and security until they generate other major accidents.

Moreover, workers take to their job all their personal characteristics such as practices, motivations, skills, attitudes, habits and personalities which can strengthen or weaken the SSMS by interacting with other working elements such as the task demands and organizational factors. Therefore, people tend not to make errors intentionally. However, we are often "set up to fail" by the way our brain processes information; training conducted, experience, skills, beliefs, the culture of the organization, the design of equipment, procedures, and so on.

Likewise, if the design and conditions (e.g. lighting, temperature) of the workplace as well as the relevant procedures are not properly managed and controlled, the employees will choose the most comfortable way to do their work, which cannot be necessarily safe [17]. Hence, any inconsistency between job needs and employees limitations and abilities rises the probability for HEs occurrence [15] which can influence, afterwards, employee health, job performance [18], as well as the safety management system [19].

The assessment of HFs and human failure management, therefore, needs to be addressed in all industrial processes. Otherwise, if certain factors combine, they will increase the number and severity of the accidents, especially in high-risk workplaces such as the energy sector, they will remain extremely astounding in terms of human and property losses [2].

\subsection{The human factors affect the SSMS}

In 1959 Heinrich stated that the identification of accidents' underlying causes ("Human Factors") during the investigations is a paramount part of accident prevention [20]. Likewise, this finding was substantiated in 1978 by Peterson; he re-emphasized that behind every accident, there are many contributing factors, causes and sub-causes [21]. Figure 2 highlights how Heinrich divided the immediate causes of preventable accidents (98\% of all accidents) into $88 \%$ of unsafe acts and $10 \%$ of unsafe conditions (mechanical or physical hazards), while, unpreventable accidents present $2 \%$ of all accidents [22].

According to Heinrich, the underlying causes can be grouped under organizational factors [20], but there are also other contributing factors, such as job factors and individual factors that constitute the HFs which affect the S-RB of the employee [18]. Figure 2 show how these factors can join randomly and cause accidents, this so-called theory of multiple causations.
A good SSMS often depends on the perfect behavior of workers in term of $H \& S$ whereas the behavior is a reflection of our knowledge, training and competence, which can be intentional or unintentional [20]. Since people's behavior represents decisions and actions taken by individuals during the cause of their daily work activities. These actions may include safe or at-risk behavior. Safe behavior defines as the behavior that supports safety practices and activities such as safety compliance in the workplace, safety leadership, safety communication, safety awareness, worker safety involvement, and so forth, which means a good SSMS in the workplace [23]. Whereas at-risk behaviors are often more suitable with employees' purposes in the workstations, they are comfortable, easier, and time saver since they are faster than safe behaviors. Consequently, the implemented $\mathrm{H} \& \mathrm{~S}$ procedures and standards will be impacted by workers unsafe acts. As well as, they are rarely upshot in negative outcomes, such as injuries or accidents.

Thereby, the SSMS is the way that workers behave [17], who sometimes do not behave safely as they are always assumed to be, due to the HFs influences. Likewise, the processes of behavior modification and values formation are the key elements to the SSMS [6].

Furthermore, the influence of the HFs in the workplace is not conditioned to the presence of $H \& S$ vulnerabilities in the SSMS of the company; however, the HFs themselves can weaken and create gaps in the SSMS. For this reason, if their impact not mitigated and eliminated at an early stage, they can be developed afterwards to an accident. Similarly, if their presence does not constitute a big risk in the workplace, they can be combined with the other factors until they become a risk sufficient to give rise to an occupational accident. Mishaps occur due to a random combination of many contributing factors [5]. Therefore, to effectively enhance the SSMS and employees' unsafe behavior, all the assessed factors and control measures should be taken into consideration during the implementation of the safety and security management system. As shown in Figure 9 and 10 the ultimate prioritization should consider the descending order from significant factors to less significant ones.

\section{OBJECTIVES AND HYPOTHESES}

This study adopts the BBS (Behavior-Based Safety) approach which intended to improve what workers do in the workplaces after analyses what and why they do in wrong way and then applies research supported control measures to improve what the workers do at the workplace and, therefore, the SSMS.

The overall aim of this study is to develop a framework of effective countermeasures to tackle the HFs that affect the SSMS in the existing and the future energy plants. To achieve these aims, the following sets of study objectives have been divided:

- to identify the impact rate of each HF on the worker behavior as well as on SSMS in the energy plants at national and international level;

- to identify commonalities of factors which affect the SSMS between the international FFPPs and ones of the focused local country, as well as, between all assessed FFPPs and wind energy;

- to identify the riskiest and the most vulnerable PPs to the influence of the HFs; 
- to introduce the appropriate control measures and a framework on HFs that promote a safe behavior from the workers which improve the SSMS in the assessed PPs;

Against this background, some hypotheses are formulated in the Table 1 to be tested in this study.

Table 1. Proposal of hypotheses for testing

\begin{tabular}{cc}
\hline $\mathbf{N}^{\circ}$ & Hypotheses \\
H1 & $\begin{array}{c}\text { "The job factors" can be the most influencing factors on } \\
\text { the SSMS of all the assessed PPs. }\end{array}$ \\
H2 & $\begin{array}{c}\text { "The workloads" can be the most influencing sub-factors } \\
\text { on the SSMS of all the assessed PPs. }\end{array}$ \\
H3 The FFPPs can be the riskiest evaluated power plants and \\
more vulnerable to the influence of the HFs than the wind \\
PPs \\
"Environment: the workplace conditions" and "Hazard and \\
Risk Perception" may have a significant relationship with \\
the human reliability as well as with SSMS. \\
H5 "Management commitment and leadership" and the \\
"Training" can have a greater effect on SSMS.
\end{tabular}

\section{MATERIALS AND METHODS}

The study attempts to improve the SSMS by identifying the interaction between the workplace environment of the energy industry and the workers' behavior, through studying those elements (Individual, job and organizational factors) which forming the HFs and causing the human failure in term of safety and security in the workplace. In other words, this proactive study assessed the impact rates of the HFs on the employees' behavioral safety compliance in a sample of Moroccan and worldwide FFPPs as well as in the Moroccan OWFs.
Figure 4 illustrate the flowchart of the methodology adopted in this study.

As previously described, those mentioned HFs affect the SSMS, through their influence on the safety behavior of the employees who can be seen through the employees' acts and actions at the workplaces in term of occupational safety.

Moreover, the data were collected through the survey questionnaire of a five-point Likert scale which has been dealing out to $\mathrm{H} \& \mathrm{~S}$ representatives of 16 FFPPs that are located across 9 countries (Morocco, Algeria, Egypt, Iraq, Iran, France, United Kingdom, Taiwan, Philippines), including 7 of whom are based in Morocco (Figure 5). Likewise, the questionnaire survey was given to the $H \& S$ representatives in the five existing operational Moroccan OWFs, which produce $47 \%$ of current wind energy. Figure 6 show the geographic location of all the assessed PPs as well as Figure 5 give an overview on the assessed PPs by groups.

In addition to the influencing factors (HFs) that allow the addressed assessors in the energy projects to express the actual impact of the HFs in their workplaces using a rating system, the survey questionnaire includes some control measures which permit the evaluators to rate the most adequate and effective measures for their sites to tackle those influencing factors of the SSMS, prior accidents occur. Afterwards, based on the revealed results, commonalities of factors which affect the process safety and security were identified between the international FFPPs and ones of the focused local country, as well as, between all assessed FFPPs and wind energy.

As shown in the Figure 3 the conducted study in the energy sector is based on factors that are associated with the individual, job and organizational factors. Those factors have an impact on the SSMS through workers behavior were identified from the OSH guidelines and relevant articles of HFs [15-18, 24-27].

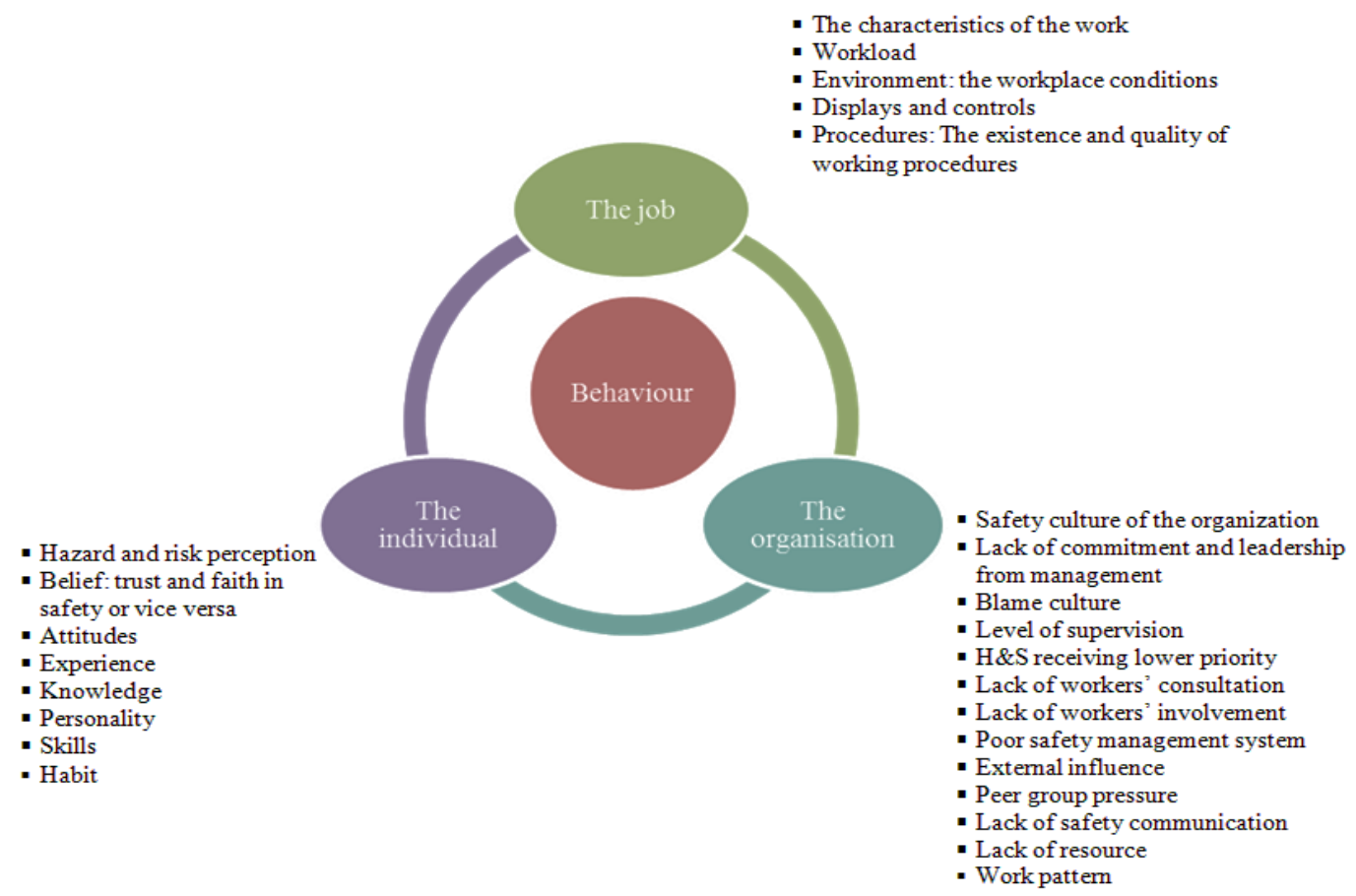

Figure 3. The human factors used in this study to improve the SSMS at the workplace 
1. Identify SSMS influencing factors via literature review.

2. Verify the inter-items consistency and reliability using SPSS

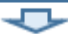

3. Design survey questionnaire to collect the information

\section{प}

4. Pilot study to highlight SSMS influencing factors in the energy industry beside the controle measures for SSMS improvement.

₹

\section{Data collection.}

\section{ए}

6. Statistical analysis. Identification of commonalities of factors which affect the SSMS in the energy sector.

\section{ए}

7. Identify the adequate control measures.

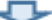

8. Communicate the outcomes of the study to all the assessed power

Figure 4. Flowchart of methodology

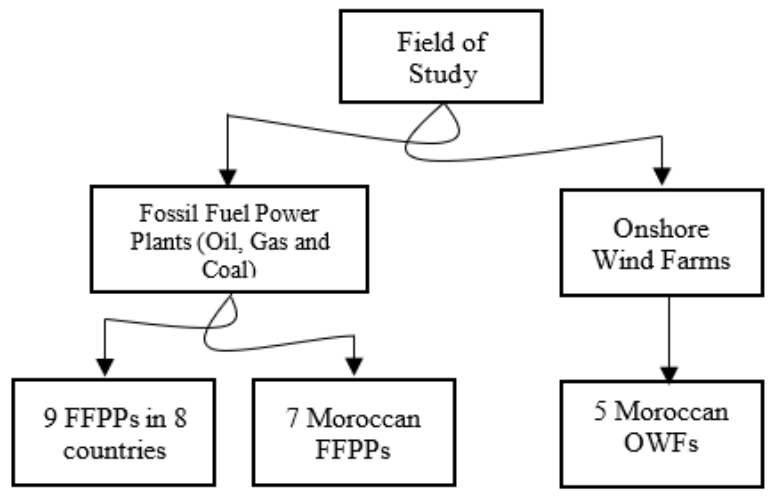

Figure 5. Field of study

The factors were selected based on their relevance to energy sector processes and work environment classified as antecedents of safety performance. Afterwards, the inter-items consistency and reliability were measured by using the coefficient of Cronbach's alpha of Statistical Package for the Social Sciences (SPSS version 20) software. In this study, Cronbach's alpha yielded 0.873 which is good and inter-items consistent.

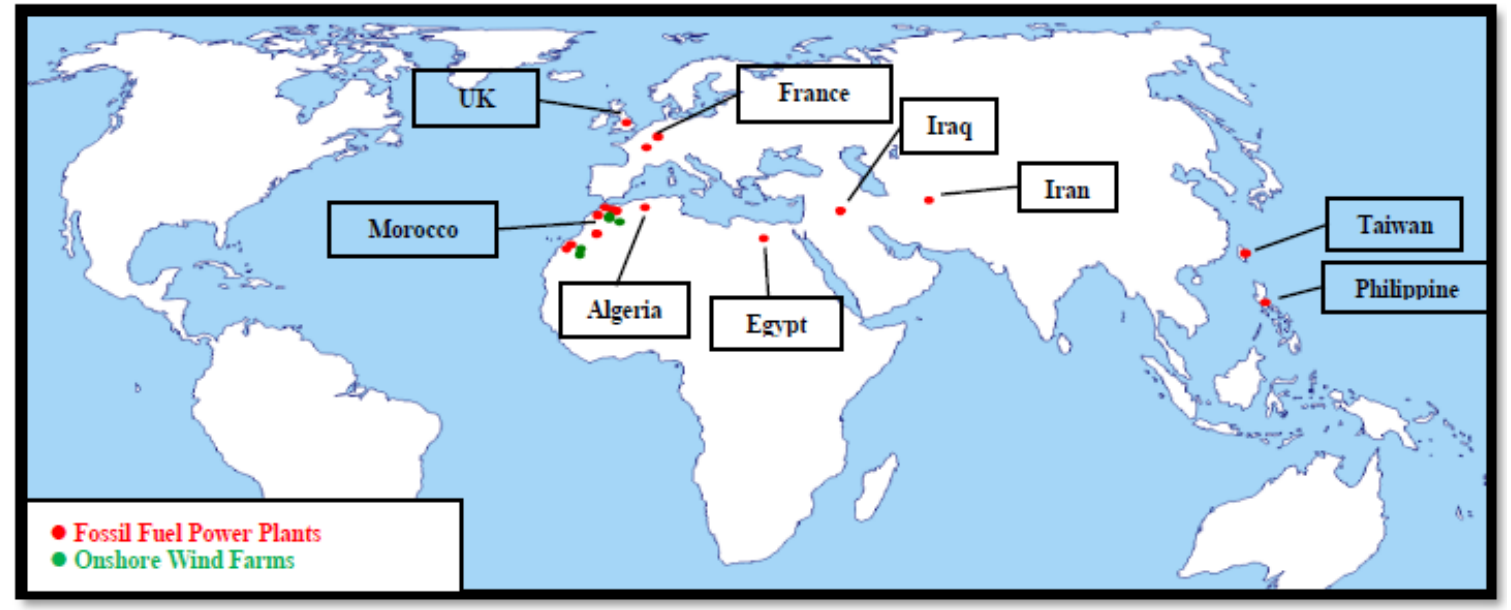

Figure 6. Geographic location of the assessed power plants

\section{RESULTS AND DISCUSSIONS}

\subsection{Factors influencing SSMS in the assessed PPs}

The bar graph of the Figure 7 show clearly that the job factors are the predominant influencing factors for all the conducted studies (3.6/5), while the individual factors come as the second affecting factors (3.4/5) and the organizational factors have the lest influence (3/5) on the SSMS within the evaluated energy plants. In other words, the job factors which entail of the nature of the task, workload, the working environment, the design of displays and controls, and the role of procedures have a greater influence on SSMS than the other assessed factors in all assessed PPs. This outcome underscores the previous finding that the nature of activity within the energy industry is deemed as high-risk activities. However, the HFs not only affects workers $H \& S$, but it also affects their productivity and efficiency.

The bar graph in the Figure 8 unveiled that the Moroccan
FFPPs are more affected by the HFs than the rest of assessed energy plants, particularly, by the job factors. Afterwards, the worldwide FFPPs are the second power generation plants influenced by the HFs, especially by the job factors again. Eventually, the OWFs are the last power generation plants that are affected by the HFs, peculiarly, by the job factors once again. In other words, the job factors are more affecting the SSMS than the other factors, especially in Moroccan FFPPs. Eventually, the study revealed that the total average of the impact rate of all the assessed HFs on the SSMS is $3.3 / 5$ on Likert scale, which is about $66 \%$ within all the evaluated energy plants.

This accordance may due to those FFPPs are riskiest assessed power generation plants and more vulnerable to the influence of the HFs than the wind industry. Nevertheless, the HFs affects the Moroccan FFPPs more than the worldwide FFPPs. Therefore, the most suffered power generation plants by the HFs, are intended to implement the introduced control measures, to improve the SSMS for accident prevention. 
Total average per each factor

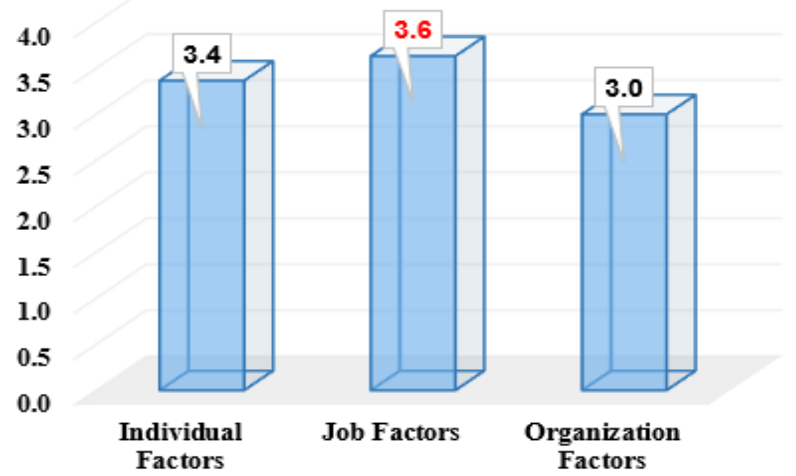

Figure 7. Overview of rating results of the assessed HFs in all the assessed PPs

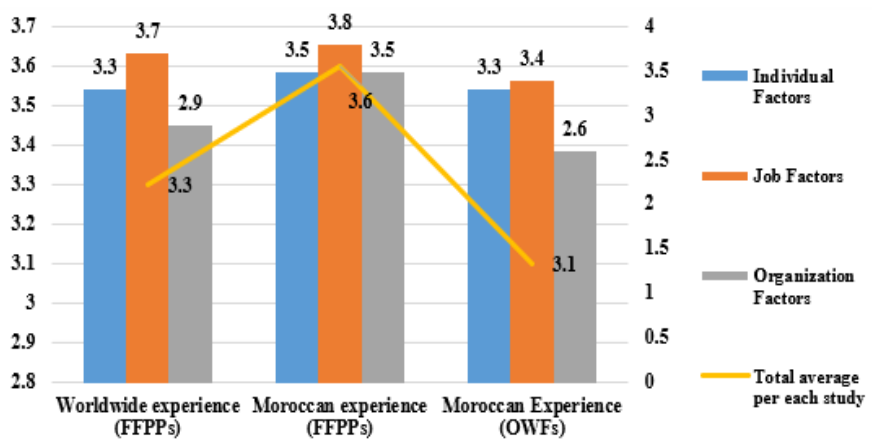

Figure 8. The impact rate of the influence of HFs on SSMS of all the evaluated PPs

The bar graph in the Figure 9 reemphasized that the "Workload" is the most affecting HF on all the assessed energy plants, particularly, by the job factors. This can be interpreted that the annual amount of maintenance works is relying basically on the age of the installed technology in PPs as well as in OWFs, hence the older the technology becomes, the more maintenance will be needed [28]. Thereby, if the management will not provide sufficient resources in term of the budget, workforce, and time for operation and maintenance of the PPs, the amount and rate of work, deadlines and variety of unplanned corrective maintenance activities that the workforce has to deal with, all will be increased. Consequently, this situation affects the SSMS through influencing the human performance in different ways, such as, human errors, burnout, the fatigue of the crew, work-related stress, health depression, workers' anxiety and time pressure.

The workload can be increased when there is an incompetent and unskilled workforce. A high workload not only affects the safety performance in the energy plants but also adversely affects job satisfaction, which, therefore, lead to an increase in the company turnover and staff shortages. For this reason, the workload should be measured if any new equipment, systems, or tasks introduced in the company processes, as well as if any alteration is made to the staff roles and responsibilities or if the case of downsizing. All the assessed FFPPs and the OWFs exceeded five years age from the commissioning time. This justifies the high rate of the workload in the workplace of the assessed energy plants, since this factor is relative basically with the age of the plant infrastructure, which after a certain period it becomes requiring more maintenance than the started time.
Likewise, some of the wind farms are lacking competent maintenance staffs, since the wind energy is a new industry in Morocco and developing progressively. Consequently, any gap in technical skills affects maintenance performance as a whole, as well as the workload will be increased, especially, some technical issues can demand more processing from inexperienced staff.

Besides, previous studies emphasized [18] that the "workload" has a significant relationship with the workers' S$\mathrm{RB}$ in the workplace, since it affects human reliability, resulting in human errors and situational violations of safety requirements in the workstation. In many cases, the "workload" is the underlying cause of numerous occupational accidents. Therefore, it necessary to tackle this underlying cause of accidents in a preventive way before injuries and accidents occur. To do so, the work responsible should consider the whole team and use a strategy when distributing tasks between team members or shifts, as well as between skilled and inexperienced workers since inexperienced staff may be less able to cope with high task demands, such as a complex maintenance task which required sufficient knowledge and experience and physical effort. Thus, task analysis should consider both physical and mental workload. Likewise, the team leader should not consider just the number of workers, but also how they are being used. In addition to this, roles and responsibilities must be set to ensure that the workforce is clear on their priorities. This will assist in ensuring that even when the workload is high, the staff will be able to concentrate on the key activities.

Furthermore, Figure 9 highlights that the "Environment: the workplace conditions" is the second HFs affect the workers S$\mathrm{RB}$ in the workplace of all the assessed energy plants. It can be possible that the "Environment: The Workplace Conditions" is a responsible factor for the "Workload" which has been earlier discussed. Figure 9 shows that these two influencing factors are the first two rated factors which are influencing the SSMS in the assessed energy plants. Likewise, the "Environment: the workplace conditions" may come from poor housekeeping in the workplace, particularly, in a multiactivity workplace that includes many workers working in different tasks which having different risks and using different tools. This situation increases the likelihood of accidents occurrence (e.g. slip, trip and fall, as well as fall of objects and so on).

As shown in Figure 9 the study revealed that the "Environment: the workplace conditions" where the workers conduct their tasks are the most affecting factor on the SSMS in the OWFs. In other words, the wind industry is a perilous workplace, since, the common wind turbine workstation is often inside the nacelle [28], which was defined in the previous study as a confined space [29] that contains various risks, such as difficult access and insufficient space for work. Similarly, the OWFs often located in remote locations where usually are lacking emergency facilities (e.g. hospitals, fire brigades, rescuers, and so on) and thus affect the process safety. Besides, the weather condition is often present a risk for workers when it windy, snowy and cold or hot environment, it affects the task and safety performance, especially, in the desert where dangerous insects (e.g. serpents, scorpions and so on) exist and posing additional risk to the workers. Likewise, those OWFs have no HSE manager who can control the SSMS at the farms, which makes the workplace conditions uncontrolled and therefore, the worker behavior will easily influence it in terms of $\mathrm{OSH}$. 


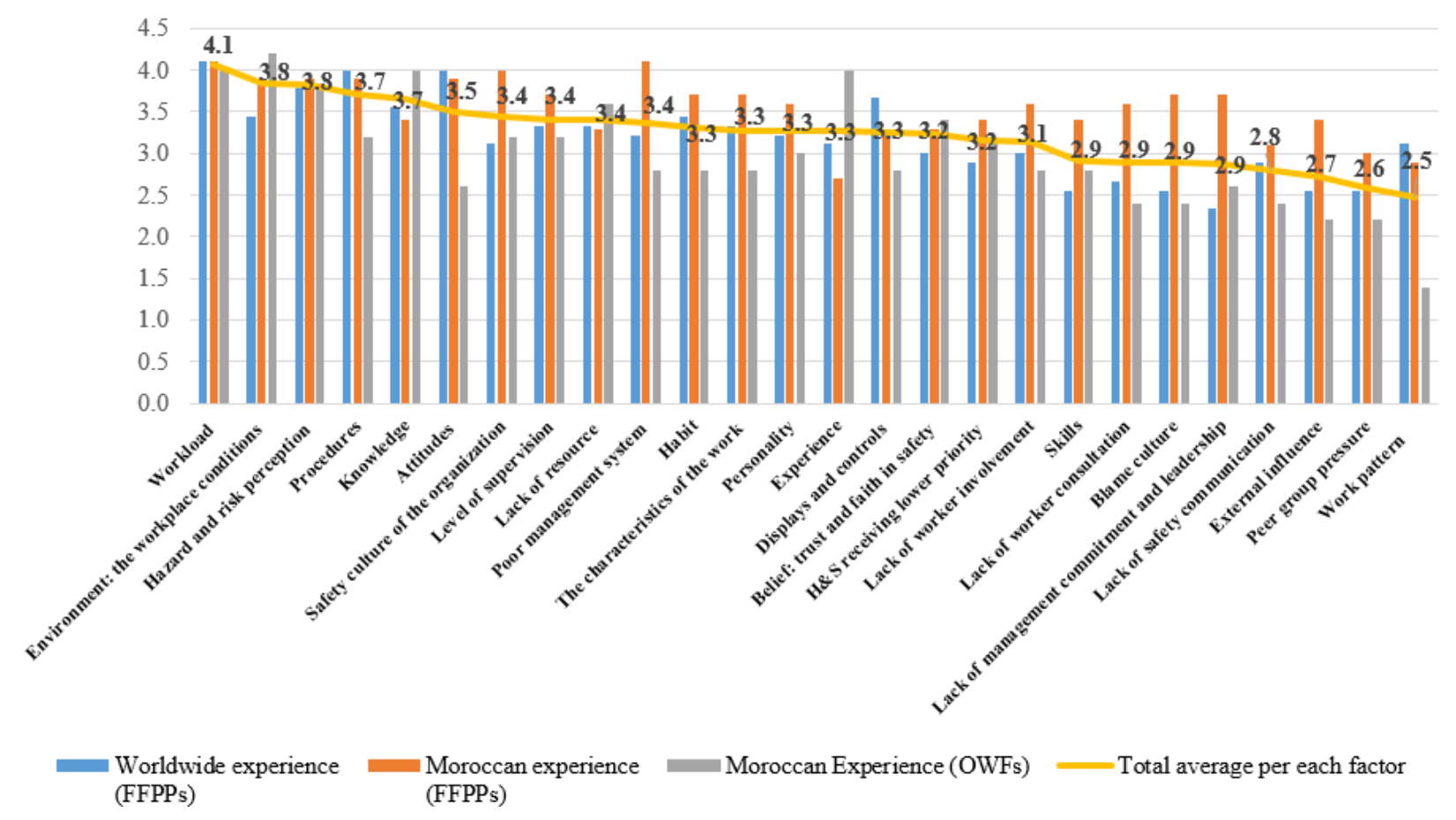

Figure 9. The average rate of the influence of each HF on the SSMS of all the evaluated PPs

Similarly, the Figure 9 presents that the "Environment: The Workplace Conditions" affects the SSMS in Moroccan FFPPs as well as the worldwide FFPPs. The difference of the impact degree between both experiences (worldwide and local FFPPs) has basically resulted from the similar implemented safety procedures in those assessed worldwide FFPPs, yet, they also have HSE managers who monitor the safety and security processes closely within those PPs, so as to mitigate the influence of the "Environment: the Workplace Conditions" on the SSMS. Whereas the Moroccan FFPPs lacks the safety management system, which makes the workplace conditions unsafe. As a result, this job factor ("Environment: The Workplace Conditions") is affected the safety performance in the workplaces of Moroccan FFPPs greater than in the worldwide FFPPs. In some cases, this factor leads to accidents [30].

The assessment in the study disclosed that the "Environment: the workplace conditions" in the FFPPs are more and less safely maintained and not present a critical safety issue, compared with the assessed OWFs. In other words, as highlighted in Figure 9 this job factor is highly impacting the safety compliance in the FFPPs workplaces than the evaluated OWFs. This may be due to the different nature of the maintenance activities that present different risks between the both evaluated power generation industry, in addition to the risks associated to the remote locations of the OWFs [8]. A former study confirmed that among the factors, the biggest influence the workers' unsafe behavior is the site condition [31].

Furthermore, the bar graph in Figure 9 clearly shows that "Hazard and Risk Perception" by the worker is the third factor that affects the SSMS in all assessed energy plants. Along the same lines, the previous study affirmed the strong relationship between risk perceptions and occupational accidents [32]. Likewise, former studies unfolded that the quality of the working environment, worker safety attitudes, job satisfaction, management involvement and commitment to H\&S, training, worker experience and competence affect their risk perceptions in the workstation [33]. Consequently, this individual factor will lead to error-producing conditions, unsafe shortcuts [34] and increase the risk of accidents [17]. Thereby, this clarified why the assigned assessors gave this individual factor much importance during filling the questionnaire, for being representing a serious factor which affecting the process safety and security of the assessed PPs.

\subsection{Control measures to enhance SSMS in the assessed PPs}

The bar graph in Figure 10 shows some control measures, which have been rated by the assessors at the energy plants, through the questionnaire survey to overcome the highlighted influencing factors and improve the safety performance in this particular sector of activity. Likewise, the graph illustrates in descending order the trends of these safety measures for each conducted empirical study, as well as the average rate per each evaluated measure. Thereby, from the bar graph in Figure 10, it is a crystal that the "Management Commitment and Leadership" is the most striking measure that has an important influence on all evaluated power generation plants to diminish or eliminate the impact of the HFs.

Afterwards, the bar graph in Figure 10 shows that the "Training" as the second essential safety measure, where the managers must invest to avoid the underlying causes of the accidents, especially, in the Moroccan FFPPs. In other words, these latter are the most needing to activate this basic safety measure so as to ensure the staffs are operating the hazardous infrastructure with a good understanding and perception of the existed hazards and risks emanated from the workplace.

Furthermore, the "BBS program" is in the third priority measure among the other control measures to be implemented at the energy plants. The BBS program analyses the HFs and their influence on the OSH performance focusing on what people do, and why they do it and then applies countermeasures to improve what people do. However, managers should regularly conduct this proactively to ensure that the employees' behavior complies with H\&S requirements. This study is basically deemed as BBS in the power generations plants. 


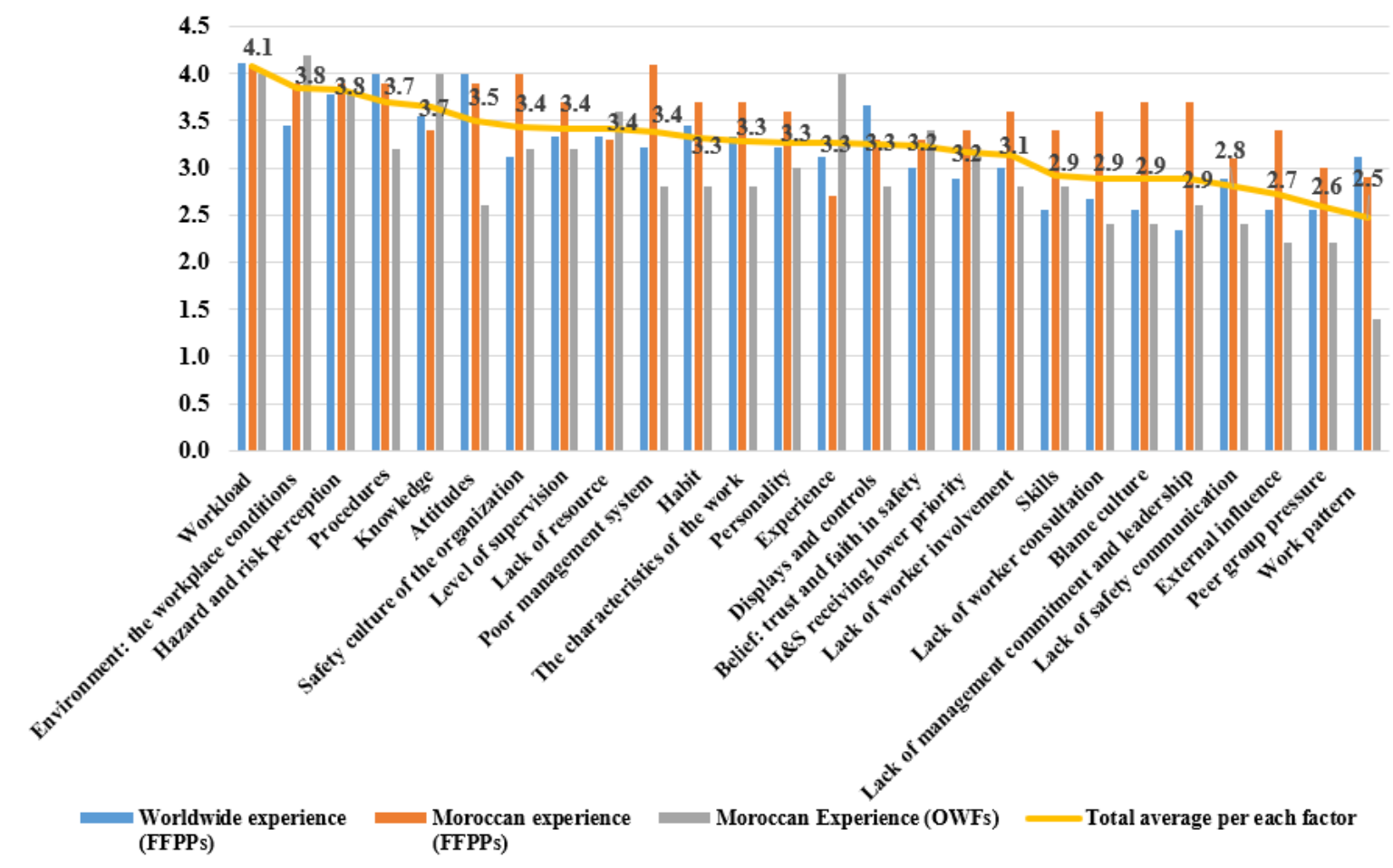

Figure 10. Control measures for the SSMS enhancement within Moroccan experience (FFPPs and OWFs) and worldwide experience (FFPPs)

Likewise, the bar graph in Figure 10 shows that the "Worker cooperation and involvement" is also the third priority measure amongst the other control measures to be implemented at the energy plants, especially, the Moroccan FFPPs which are the most needing to put in place this safety measure to enhance their SSMS. The used rating system in the survey questionnaire gives a logical sequence of the measures. It should be implemented within the Moroccan FFPPs by importance order, starting by the training of the staffs, then they should be involved and cooperated in the H\&S processes to enhance the safety performance by implementing this safety knowledge and practice them in day to day business. This upshot will form a competent staff, which they will enhance the safety and security culture of the energy plant.

The bar graph in Figure 10 substantiates that the Moroccan energy plants are the first PPs group giving high rates almost to all the assessed control measures. This gives the assumption that PPs the local FFPPs are the most needed to implement all the introduced control measures to improve their SSMS. While, the FFPPs of the worldwide experience are the second PPs group giving low rates for all the evaluated control measures, after the Moroccan FFPPs. Finally, the local wind farms are the last PPs group which give the lowest rate for all the proposed control measures.

The most remarkable result to emerge from the data is shown in Figure 10; that the obtained rating results of the control measures to be implemented in the PPs are confirming the results which displayed in Figure 8. In other words, the more a PPs group is impacted by the HFs the more it giving a high rate of the control measures to be implemented to enhance their SSMS for accidents prevention. Thus, the local FFPPs are the most affected by the HFs. Afterwards, the worldwide FFPPs and then the OWFs comes as the last energy plants least influenced by the HFs. Therefore, the local FFPPs are the riskiest assessed energy plants and they need to implement the control measures to minimise the HFs impact as well as to improve their SSMS.
Besides, all the proposed hypotheses of this study are accepted as shown in the Table 2.

Table 2. Summary of the tested hypotheses

\begin{tabular}{cc}
\hline Hypothesis $\mathbf{N}^{\circ}$ & Finding \\
\hline $\mathrm{H}^{1}$ & Accepted \\
$\mathrm{H}^{2}$ & Accepted \\
$\mathrm{H}^{3}$ & Accepted \\
$\mathrm{H}^{4}$ & Accepted \\
$\mathrm{H}^{5}$ & Accepted \\
\hline
\end{tabular}

\section{CONCLUSION}

To ensure a comfortable life that the energy industry provides to the human society and the boost that it gives to the world economy, this paper studying the effect of the underlying causes of the majority of the occurred accidents (HFs) on the SSMS in this hazardous industrial sector. This study is shed new light on the development of the safetyrelated behaviour model for national and international setting in both types of energy power generation (OWFs and FFPPs) involving developing and developed countries. Therefore, this research has important guiding significance to prevent PPs accidents through improving workers unsafe behaviours contributing to injuries and losses, as well as, increasing the energy availability and efficiency the of the PPs. The study has concluded that all the assessed HFs have influenced the workers S-RB and, therefore, the SSMS of all the evaluated energy plants with an impact rate of $3.3 / 5$ on Likert scale. Moreover, a good SSMS can be achieved in the assessed PPs if the job factors are improved, especially in the Moroccan FFPPs. Similarly, if the employees' "Workload" is well managed in the workplace, as well as if the management clearly demonstrates their commitment and leadership in dayto-day business, also they will enhance the SSMS in all the 
assessed power generation plants. Likewise, the study revealed that the SSMS of the fossil PPs are the most affected by the HFs particularly, Moroccan FFPPs, which make, subsequently, this PPs group riskier and more vulnerable to undesired outcomes that may arise from uncontrolled HFs. Previous studies corroborated that Morocco has alarming statistics in terms of occupational accidents [8-10]. Therefore, the FFPPs especially the Moroccan fossil PPs are more intended to implement the control measures from the most to the least significant measure to enhance effectively the SSMS. In the same vein, the results of the study can be employed to enhance the SSMS in the energy industry as a whole or effectively a particular energy sector (Fossil or wind energy plants) as well as to improve the SSMS in developing countries similar to Morocco where the energy sector is still growing. It's noteworthy to state that these influencing factors are not only applicable to the energy industry but also other industries. Hence, the analyzed HFs in this study to enhance the SSMS in the power generation plants are spur to future study in other hazardous industries such as manufacturing and construction industries where many accidents occur [35].

\section{ACKNOWLEDGEMENT}

The authors would like to thank all those who agreed to support this study and gave generously of their time in doing so.

\section{REFERENCES}

[1] Ünsar, A.S., Süt, N. (2015). Occupational accidents in the energy sector: Analysis of occupational accidents that occurred in thermal and hydroelectric centrals between 2002 and 2010 in Turkey. Procedia - Social and Behavioral Sciences, 181: 388-397. https://doi.org/10.1016/j.sbspro.2015.04.901

[2] Hirschberg, S., Spiekerman, G., Dones, R. (1998). Project GaBE: Comprehensive assessment of energy systems. Severe Accidents in the Energy Sector, Paul Scherrer Institut, 98-116.

[3] Fritzsche, A.F. (1989). The health risks of energy production. Risk Analysis, 9(4): 565-577. https://doi.org/10.1111/j.1539-6924.1989.tb01267.x

[4] Chen, D., Tian, H. (2012). Behavior based safety for accidents prevention and positive study in China construction project. Procedia Engineering, 43: 528-534. https://doi.org/10.1016/j.proeng.2012.08.092

[5] Choudhry, R.M. (2008). Why operatives engage in unsafe work behavior: Investigating factors on construction sites. Safety Science, 46(4): 566-584. https://doi.org/10.1016/j.ssci.2007.06.027

[6] Khan, M., Khan, H.J. (2003). Determinant of occupational safety and health performance in small and medium manufacturing setting. Masters thesis, Universiti Utara Malaysia, Kedah Darul Aman, Malaysia.

[7] Rohani, J.M., Johari, M.F., Hamid, W.H.W., Atan, H., Adeyemi, A.J., Udin, A. (2015). Occupational accident direct cost model validation using confirmatory factor analysis. Procedia Manufacturing, 2: 286-290. https://doi.org/10.1016/j.promfg.2015.07.050

[8] El Kholti, A. (2015). Stratégies pour la sécurité et la santé au travail. https://docplayer.fr/12209153-Sante-securiteau-travail.html.
[9] Hämäläinen, P., Takala, J., Saarela, K.L. (2005). Global estimates of occupational accidents. Safety Science, 44(2): 137-156. https://doi.org/10.1016/j.ssci.2005.08.017

[10] Omar, H.A. (2017). Occupational safety in Morocco and ministry of labour.

[11] Atombo, C., Wu, C., Tettehfio, E.O., Nyamuame, G.Y., Agbo, A.A. (2017). Safety and health perceptions in work-related transport activities in ghanaian industries. Safety and Health at Work, 8(2): 175-182. https://doi.org/10.1016/j.shaw.2016.10.002

[12] Tucker, S., Diekrager, D., Turner, N., Kelloway, E.K. (2014). Work-related injury underreporting among young workers: Prevalence, gender differences, and explanations for underreporting. Journal of Safety Research, 50: 67-73. https://doi.org/10.1016/j.jsr.2014.04.001

[13] Wachter, J.K., Yorio, P.L. (2014). A system of safety management practices and worker engagement for reducing and preventing accidents: An empirical and theoretical investigation. Accident Analysis and Prevention, 68: 117-130. https://doi.org/10.1016/j.aap.2013.07.029

[14] Ünal, Ö., Akbolat, M., Amarat, M., Tilkilioğlu, S. (2018). The role of the human factor in occupational safety and health performance. International Journal of Occupational Safety and Ergonomics, 1-17. https://doi.org/10.1080/10803548.2018.1554932

[15] Lorenzo, D.K., P.E., EQE, I.I. (2001). Reducing Human Errors. Washington D.C., USA. https://documents.pub/document/api-770-reducinghuman-errors-2001.html.

[16] Human error risk reduction to ALARP. National Offshore Petroleum Safety and Environmental Management Authority. https://www.nopsema.gov.au/assets/Informationpapers/A424182.pdf.

[17] National General Certificate Unit. (2015). NEBOSH certificate courses - sample material. National Examination Board in Occupational Safety and Health, 978.

[18] Health and Safety Executive. (1989). Reducing error and influencing https://www.hse.gov.uk/pubns/priced/hsg48.pdf

[19] Skeepers, N.C., Mbohwa, C. (2015). A study on the leadership behaviour, safety leadership and safety performance in the construction industry in South Africa. Procedia Manufacturing, 4: 10-16. https://doi.org/10.1016/j.promfg.2015.11.008

[20] Mihai, M.P., Sorin, B.M. (2015). Behavioral based safety management in electric power industry-complementary safety of workers. Behavioral Based Safety Management in Electric Power Industry, 7: 303-307.

[21] Health and Safety Executive. (2017). Accident aetiology lecture notes. https://www.hse.gov.uk/quarries/education/index.htm.

[22] Manuele, F.A. (2011). Dislodging two myths from the practice of safety. Professional Safety, 56(10): 52-61.

[23] Andriessen, J.H.T.H. (1978). Safe behaviour and safety motivation. Journal of Occupational Accidents, 1(4): 363-376. https://doi.org/10.1016/0376-6349(78)90006-8

[24] Gordon, R.P.E. (1998). The contribution of human factors to accidents in the offshore oil industry. Reliability Engineering and System Safety, 61(1-2): 95- 
108. https://doi.org/10.1016/S0951-8320(98)80003-3

[25] Bryden, R.N. (2006). The organisational antecedents of individual safety behaviour in the U.K. offshore oil and gas industry. Ph.D. thesis. University of Aberdeen, Aberdeen, UK.

[26] Borghini, F. (2018). Human factor analysis inside a peculiar job environment at the Gran Sasso Mountain underground laboratory of Italian national institute for nuclear physics. International Journal of Safety and Security Engineering, 8(3): 390-405. https://doi.org/10.2495/SAFE-V8-N3-390-405

[27] Summers, A., Roche, E. (2020). A practical approach to preventing systematic error in the maintenance of instrumented safeguards. Process Safety Progress, 39(2): 1-11. https://doi.org/10.1002/prs.12102

[28] Chaumel, J., Ilinca, A., Laurent, G. (2015). Wind energy secto occupational health and safety risks and accident prevention strategies. Bibliothèque et Archives nationales du Québec.

[29] Aneziris, O.N., Papazoglou, I.A., Psinias, A. (2016). Occupational risk for an onshore wind farm. Safety Science, $\quad 88$ : 188-198. https://doi.org/10.1016/j.ssci.2016.02.021

[30] Zhang, Y., Shao, W., Zhang, M., Li, H., Yin, S., Xu, Y. (2016). Analysis 320 coal mine accidents using structural equation modeling with unsafe conditions of the rules and regulations as exogenous variables. Accident
Analysis and Prevention, 92: 189-201. https://doi.org/10.1016/j.aap.2016.02.021

[31] Mohammadfam, I., Ghasemi, F., Kalatpour, O., Moghimbeigi, A. (2017). Constructing a Bayesian network model for improving safety behavior of employees at workplaces. Applied Ergonomics, 58: 3547. https://doi.org/10.1016/j.apergo.2016.05.006

[32] Clarke, S.G. (2000). Safety culture: Under- specified and overrated? International Journal of Management Reviews, 2(1): 65-90. https://doi.org/10.1111/14682370.00031

[33] Mearns, K., Flin, R. (1995). Risk perception and attitudes to safety by personnel in the offshore oil and gas industry: A review. Journal of Loss Prevention in the Process Industries, 8(5): 299-305. https://doi.org/10.1016/09504230(95)00032-V

[34] Reason, J. (1995). Understanding adverse events: Human factors. Quality in Health Care, 4: 80-89. https://doi.org/10.1136/qshc.4.2.80

[35] Khosravi, Y., Asilian-Mahabadi, H., Hajizadeh, E., Hassanzadeh-Rangi, N., Bastani, H., Behzadan, A.H. (2014). Factors influencing unsafe behaviors and accidents on construction sites: A review. International Journal of Occupational Safety and Ergonomics, 20(1): 111-125.

https://doi.org/10.1080/10803548.2014.11077023 